\title{
Lattice-Based Walks and the Monte Carlo Method for Addressing Mass, Thermal and Elasticity Problems
}

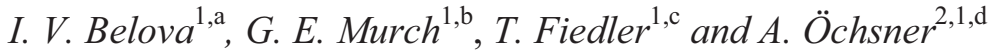

${ }^{1}$ Centre for Mass and Thermal Transport in Engineering Materials, Priority Research Centre for Geotechnical and Materials Modelling,

School of Engineering,

The University of Newcastle

Callaghan, New South Wales 2308, Australia

${ }^{2}$ Department of Applied Mechanics, Faculty of Mechanical Engineering, Technical University of Malaysia, 81310 UTM Skudai, Johor, Malaysia

aIrina.Belova@newcastle.edu.au, 'raeme.Murch@newcastle.edu.au, cThomas.Fiedler@newcastle.edu.au, ${ }^{\mathrm{d}}$ Andreas.Oechsner@gmail.com

Keywords: Monte Carlo, diffusion, thermal conductivity, bulk modulus

\begin{abstract}
In this paper, we review the recent developed method based around latticebased random walks and the Monte Carlo method. This method, which is now called the Lattice Monte Carlo method, permits complex phenomenological problems in diffusion, thermal conductivity and elasticity to be addressed. It is shown how the effective mass diffusivity, thermal diffusivity/thermal conductivity and the bulk modulus in composites can be calculated and also how concentration profiles and temperature profiles can be determined in situations where the diffusivity depends on position and concentration and the thermal conductivity depends on position and temperature respectively.
\end{abstract}

\section{Introduction}

The Monte Carlo method has long been used to address mass and thermal transport problems. For many years, mass transport problems were treated purely at an atomic level. Many of the early calculations, especially in the 1960s, used the Monte Carlo method purely as a semi-quantitative way for visualizing such kinetic processes as alloy ordering kinetics and radiation damage annealing. Later, in the 1970s, many of these atomistic Monte Carlo calculations became much more quantitative through the determination of tracer and collective correlation factors and average jump frequencies notably in alloys and high defect solids. In 1983, making use of a general 'lattice model' that was originally devised for atomic motion in and around a grain boundary [1], the Monte Carlo method was extended to much longer length scales by simply removing the nexus between the lattice and atomic jumps [2]. This now enabled phenomenological diffusion problems to be addressed by simply mapping a lattice or grid onto the phenomenological problem. The lattice thus lost its atomic significance. The lattice was simply explored by non-interacting virtual particles that hopped at rates that were simply proportional to the diffusivities in the problem. In essence, this was a finite difference method making use of the Monte Carlo method and a lattice. This method has been called the Lattice Monte Carlo 
(LMC) method to separate it conceptually from atomistic Monte Carlo calculations. The latter are now generally called Kinetic Monte Carlo (KMC) calculations.

In this review paper, we present LMC procedures for calculating 1) the effective diffusivity and concentration profiles, 2) effective thermal conductivities, thermal diffusivities and temperatures profiles, and finally 3 ) the effective bulk modulus.

\section{The Effective Diffusivity.}

The effective diffusivity refers, for example, to the effective diffusivity of a microcrystalline material where, in a single phase material, it is in general an unknown weighted average of the bulk diffusivity and the diffusivity along grain boundaries as measured, say, in the Harrison Type-A kinetics regime [3]. Or it could be the effective diffusivity of a diffusant in a two-phase material [4] where again it is an unknown weighted average of the diffusivities of a diffusant in each of the two phases, and might also, of course, include contribution from diffusion along the interphase boundaries. The nature of the weighted average depends on the size, shape and orientation of the various diffusion regions, in other words their geometry. If the diffusion regions are parallel to the diffusion direction then the effective diffusivity is given exactly by a simple weighted arithmetic mean (the Hart equation [5]). If the different diffusion regions alternate in the diffusion direction, then the effective diffusivity is given exactly by a simple weighted geometric mean.

In a LMC calculation, it is best by far to calculate the effective diffusivity directly from the Einstein-Smolochowski (ES) Equation. However, it is also possible to calculate the effective diffusivity by the LMC method by making direct use of Fick's first law under steady-state conditions or by making use of Fick's second law by processing a simulated concentration profile in much the same way an experimental (tracer) concentration profile might be processed.

The ES equation, also often simply called the Einstein equation, describes the self-diffusivity $D$ of randomly walking particles in $d$ dimensions $(d=1,2,3)$ :

$$
D=\frac{<R^{2}>}{2 d t}
$$

where $R$ is the vector displacement of a given exploring particle after some long time $t$ and the Dirac brackets $<>$ refer to an average over a very large number $(N)$ of particles. The ES Equation refers only to a system already at equilibrium. In a mass diffusion context, the ES Equation refers then to the determination of the diffusivity of individual particles that can be followed or 'traced' in a system that is already at chemical equilibrium, i.e. with no concentration gradient acting or external field(s) acting. The individual diffusivities in each region are described by the ES equation as well as the effective diffusivity of the whole material. For random walks without correlations on a simple cubic lattice the diffusivity $D_{i}$ in each region $i$ can be partitioned from Eq. (1) as:

$$
D_{i}=\frac{\Gamma_{i} r^{2}}{6}
$$

where $\Gamma_{i}$ is the site to site jump rate and $r$ is the jump distance (the distance between sites). A suitably fine-grained simple cubic lattice is overlaid on the 
phenomenological problem. The different diffusivities in the problem are then simply represented as different jump frequencies on the latttice.

In contrast to the other principal diffusion kinetics simulation method of molecular dynamics, simulation of real time is not possible in a Monte Carlo kinetics calculation. What one actually does is to use a discrete quantity that is proportional to real time. This quantity is the number of jump attempts per particle. Using the ES Equation with this quantity acting as 'time', one can then calculate a relative diffusivity, i.e. a diffusivity that is relative to one of the specified diffusivities (usually the highest) in the system. In contrast to atomistic Monte Carlo calculations, in a LMC calculation there are no correlation effects in the diffusion of the particles: each particle diffuses completely independently of all others. Put another way, multiple occupancy of a lattice site is always permitted. Particles can be released one at a time and allowed to diffuse for a time $t$ or they can be released simultaneously and then allowed to diffuse for a time $N t$. The lattice is normally made periodic i.e. the basic structure is surrounded by images of itself, but the displacements $R$ are calculated by ignoring the boundaries. The time needs to be sufficiently long that the effective diffusivity of the material has converged.

In many materials, one can expect that a diffusant will undergo segregation among the various regions that are present. For two-phase material for example, this is conveniently expressed through a segregation factor $s$ which is usually defined by way of Henry's Law as [6]:

$$
s=\frac{c_{2}}{c_{1}}\left(=\exp \left(-\frac{E_{s}}{k T}\right)\right)
$$

where, for convenience, $c_{1}<c_{2}$ where $c_{\mathrm{i}}$ is the (equilibrium) concentration of the diffusant in phase i. In Eq. (3), $E_{\mathrm{s}}$ is the segregation energy of the diffusant between the two phases, $k$ is the Boltzmann constant and $T$ is the absolute temperature. In the implementation of segregation in the LMC method, the jump probability $\Gamma_{12}$ for a jump from phase 1 to phase 2 and the jump probability $\Gamma_{21}$ for a jump from phase 2 to phase 1 are related to the segregation factor at equilibrium by:

$$
\Gamma_{12} / \Gamma_{21}=s
$$

When segregation is present, the initial release of the particles must also be adjusted. This is simply achieved by weighting the regions according to Eq. (3) and their relative fractions.

In some cases, it is appropriate to take into account blocking effects of the diffusant in each diffusion region, in which case, for a two-phase material, the McLean isotherm is used [6].

$$
s=\frac{C_{2}\left(1-C_{1}\right)}{C_{1}\left(1-C_{2}\right)}=\exp \left(-\frac{E_{s}}{k T}\right)
$$

The jump frequencies $\Gamma_{12}$ and $\Gamma_{21}$ would then be adjusted according to the number of particles on that site. 
Most of the LMC calculations of the effective mass diffusivity thus far have focussed on the effective diffusivity when there are contributions from diffusion in the bulk and diffusion along grain boundaries, e.g. [7-10] and references therein.

\section{Determination of Concentration Profiles.}

Obtaining the concentration profile using the LMC method is equivalent to solving the Diffusion Equation (Fick's second law) for a problem once initial and boundary conditions have been designated. For example, consider the well-known experimental situation where a very thin layer of tracer (the diffusant) is deposited at the surface of the material at time $t=0$ (This is sometimes referred to as the thin-film or instantaneous source condition). The tracer is then permitted to diffuse for some diffusion time $t$. A source plane of particles is established in the centre of a large periodic simple cubic lattice in order to avoid edge effects. Several million particles are generated at random positions on this source plane and released either sequentially or simultaneously. Assuming the latter for convenience here, the particles are allowed to diffuse independently of one another for the entire time $N t$, at which time the final position of each particle is recorded. The final positions of all of the particles are then simply assembled to form a concentration penetration profile. Provided the lattice is large in the diffusion direction, at relatively short times, the profile corresponds to the familiar Gaussian profile as appropriate for diffusion into a 'semi-infinite' solid, see Fig. 2. If a periodic boundary is in place in the diffusion direction and the two halves separated at the source plane after diffusion then this corresponds to diffusion into a thin material from both sides.

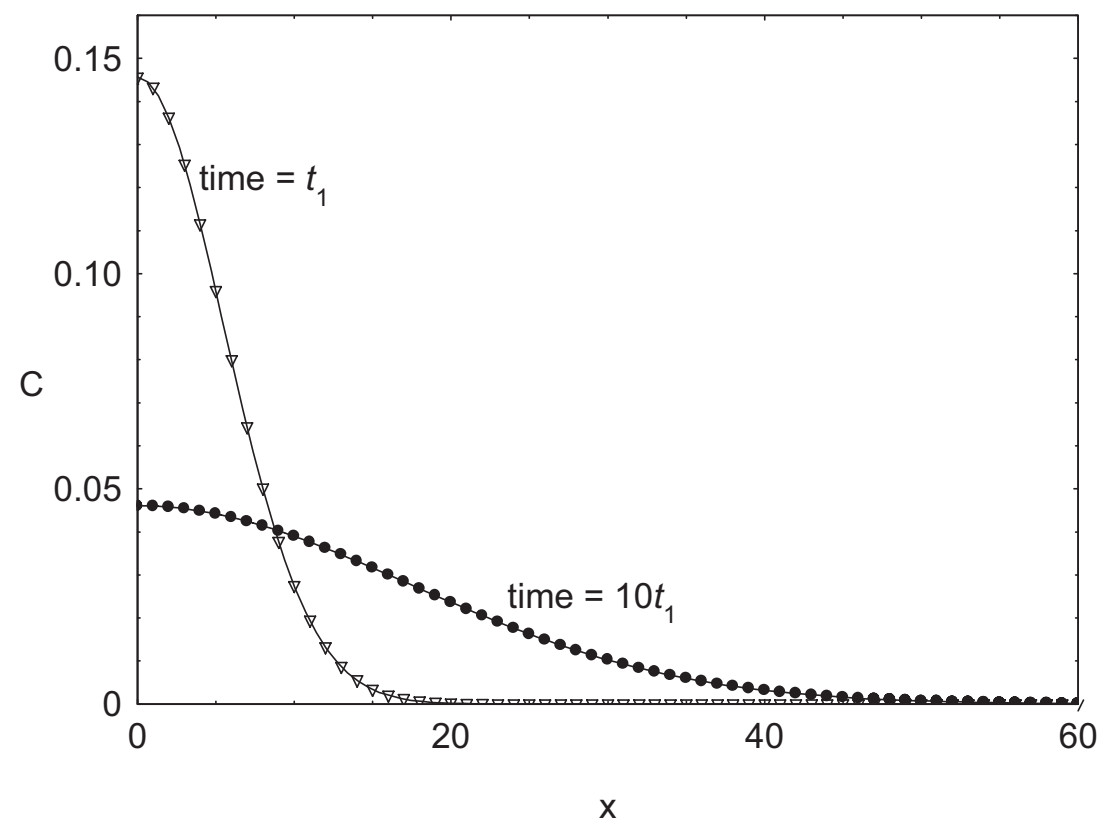

Fig. 2. Typical Gaussian concentration profiles for tracer diffusion from an instantaneous source at $\mathrm{x}=0$ for two different times (symbols represent LMC results).

In many diffusion problems, the diffusivity is a (known) function of concentration. This is very readily incorporated into the LMC method and the corresponding concentration profile determined. The method uses the basic procedure 
in which all particles are released simultaneously. Since the jump rate is directly proportional to the diffusivity at each jump attempt, the number of particles on a given site at that time is simply noted (this is the local concentration) and this is then used via the known $D(C)$ to rescale the jump rate at that site.

Most of the applications of the LMC method to calculate concentration profiles have so far been in the context of diffusion in the presence of grain boundaries; see, e.g. $[11,12]$.

\section{Determination of the Effective Thermal Diffusivity and Thermal Conductivity}

The diffusion of heat, like mass, is a random process that can also be represented by random walks of particles that can be considered as virtual 'heat particles'. The ES equation also describes the dispersion of the thermal diffusivity $K$ in $d$ dimensions ( $d$ $=1,2,3)$ :

$$
K=\frac{<R^{2}>}{2 d t}
$$

It should be noted that the thermal conductivity $\lambda_{i}$ in a phase $i$ is directly related to the thermal diffusivity $K_{\mathrm{i}}$ in that phase by the well-known expression $K_{\mathrm{i}}=\lambda_{\mathrm{i}} / \rho_{i} C_{p, i}$ where $\rho_{i}$ is the density of phase $i$ and $C_{p, i}$ is its specific heat. In a model composite, by simply requiring that the densities and the specific heats take values (hypothetically) equal to unity everywhere in the calculation, the effective thermal conductivity $\lambda_{\text {eff }}$ then simply equals the effective thermal diffusivity $K_{\text {eff. }}$ The ES Equation as used here has no physical meaning in the sense that it can be made use of experimentally. It purely provides a useful means for calculating, in models, the effective thermal diffusivity (and thermal conductivity) from random walks of virtual heat particles.

The general procedure above for the calculation of the effective mass diffusivity applies equally to the calculation of the effective thermal conductivity. Accordingly, all the results for the various models that have been obtained for the effective mass diffusivity apply equally to the effective thermal conductivity. Several calculations have been specifically directed to the LMC calculation of the effective thermal conductivity in models of composites [13]. In addition, there have been calculations of the effective thermal conductivity of models of syntactic metallic hollow sphere structures, usually known as MHSS materials [15] and compact heat sinks based on cellular metals [16]. An example of the results of a LMC calculation of the effective thermal conductivity is shown in Fig. 3 for the case of circular inclusions in a matrix [13]. In the same figure are also the results for the effective thermal conductivity using finite element analysis. The agreement is seen to be excellent.

There have been no direct LMC calculations of the effective thermal diffusivity. However, the effective diffusivity can be readily formed from the effective thermal conductivity calculated by LMC using the following equation:

$$
K_{\text {eff }}=\frac{\lambda_{\text {eff }}}{\left(\rho C_{p}\right)_{\text {eff }}}
$$

where

$$
\left(\rho C_{p}\right)_{\text {eff }}=\sum_{\text {all phases } i} \rho_{i} C_{p, i} g_{i}
$$




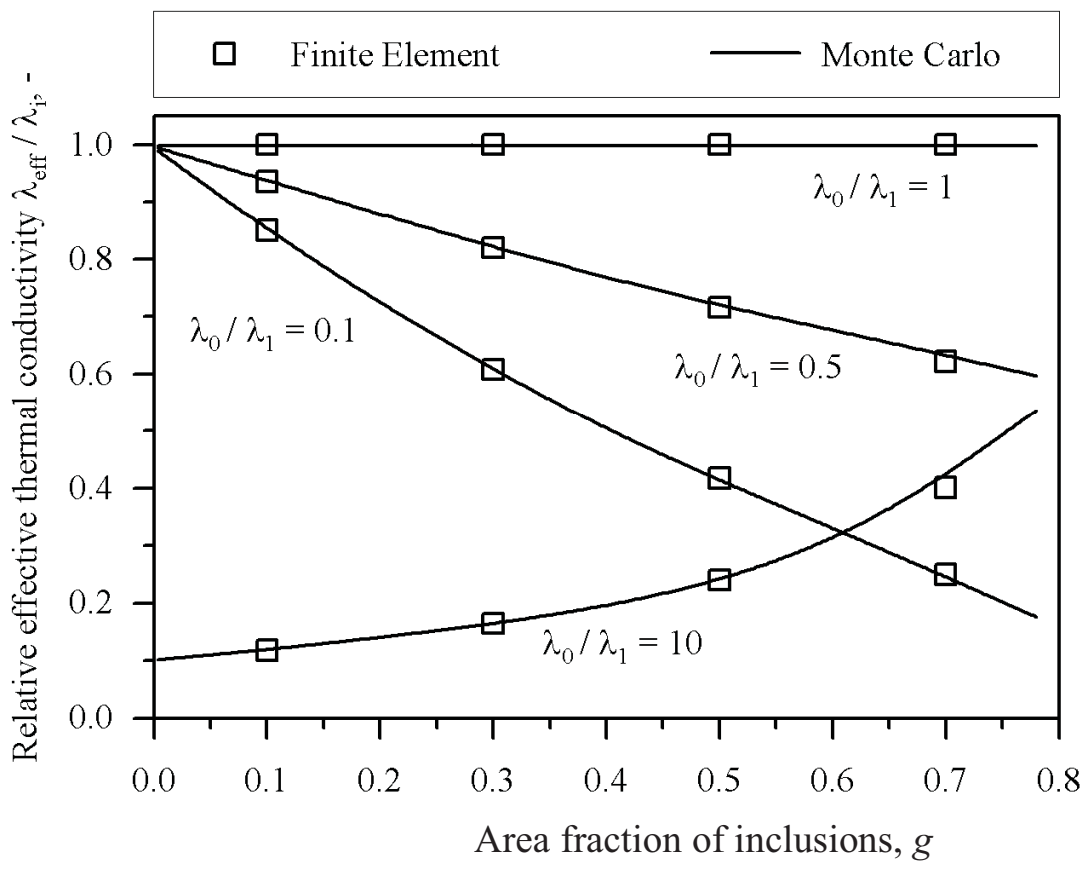

Fig. 3. Comparison of results from LMC and Finite Element Method calculations of the relative effective thermal conductivity of a composite with circular inclusions (0) in a matrix (1) in a square planar arrangement as a function of area fraction of inclusions for several values of the matrix and dispersed phase thermal conductivities.

\section{Determination of Temperature Profiles.}

Because of the role of the density and specific heat in transient heat transport problems, the determination of temperature profiles requires a different computational procedure from that described above for concentration profiles. We will describe it in a little detail. We will focus on the case where the temperature at the surface or source is held constant at $T_{\mathrm{c}}$ and we will determine the temperature profile in the composite material. The number of virtual heat particles in the source plane is $N_{\mathrm{n}}$. The amount of thermal energy $E_{\mathrm{p}}$ corresponding to a virtual thermal energy particle is given by the relation:

$$
E_{p}=T_{\mathrm{c}} \cdot r^{3} \cdot \rho \cdot C_{p} \cdot \frac{1}{N_{\mathrm{n}}}
$$

The random walks of the particles on the lattice are now organized in such a way that they are directed by two parameters: the jump probability $p_{\mathrm{j}}$ (the scaled thermal conductivity) and the selection probability $p_{\mathrm{s}}$ (the scaled inverse product $\rho_{i}$ - $\left.C_{\mathrm{p}, i}\right)$. The selection probability is treated as an 'amount of inertia' assigned to a virtual thermal particle in the particular phase: i.e. the higher the specific heat is in a phase the slower the virtual thermal particle. Both jump and selection probabilities depend on the material parameters of the phase $i$. The jump and selection probabilities $p_{\mathrm{j}}$ and $p_{\mathrm{s}}$ are defined to have values between zero (the event never occurs) and unity (the event always occurs). Then the jump probability in a phase must be scaled with respect to the highest thermal conductivity so that: 


$$
p_{\mathrm{j}, i}=\lambda_{i} / \lambda_{\max }
$$

And the selection probability $p_{s, i}$ is scaled with respect to the lowest value of the product $\rho_{i} \cdot C_{\mathrm{p}, i}$ :

$$
p_{s, i}=\frac{\left(\rho_{j} \mathrm{C}_{p, j}\right)_{\min }}{\rho_{i} \mathrm{C}_{p, i}}
$$

Thus according to Eq. (10), a phase with a high specific heat and density possess a low selection probability. Different selection probabilities between sites from different material regions results in the modified (Eq. (10)) definition of the energy $E_{\mathrm{p}}$ corresponding now to a virtual heat particle:

$$
E_{p}=T_{\mathrm{c}} \cdot r^{3} \cdot\left(\rho \cdot C_{p}\right)_{\min } \cdot \frac{1}{N_{\mathrm{n}}}
$$

Let us assume that phase 1 has a lower selection probability than phase 2 i.e.: $p_{1}<p_{2}$. The overall probability of a jump of a probing heat particle in phase 1 is then equal to $p_{\mathrm{s}, 1} \cdot p_{\mathrm{j}, 1}$ and similarly for a particle in phase 2 (This value is, in fact, a scaled thermal diffusivity). The increased number of unsuccessful jump attempts in phase 1 simulates an accumulation of virtual heat particles in that phase. It should be mentioned here that the selection of $p_{\mathrm{s}}$ and jump probabilities $p_{\mathrm{j}}$ of virtual heat particles inside the source plane $(x=0)$ are equal to unity.

At the beginning of each time-step, a particle is randomly selected. Next, a random number between 0 and 1 is generated and compared to the selection probability corresponding to the phase at that lattice site. If this random number is higher than the selection probability, the attempt is unsuccessful; the LMC time is increased and another particle is randomly chosen. Otherwise, a jump direction for the particle is randomly chosen and, depending on the phase(s) of the starting and target lattice sites, the jump probability $p_{\mathrm{j}}$ is now determined. In the case that the jump attempt is successful, the coordinates of the particle are updated before the LMC time is increased and a new particle is selected. The incremental increase to the Monte Carlo time $t_{\mathrm{MC}}$ depends on the total number $N_{\text {ges }}$ of virtual heat particles in the system. At the end of the specified time $t_{\mathrm{MC}}$ the final positions of all of the particles are recorded.

The results of the LMC analyses are virtual heat particle distributions that correspond to particular Monte Carlo times $t_{\mathrm{MC}}$. In order to obtain temperature profiles, the heat particles are translated into site temperatures $T$ according to:

$$
T=\frac{n \cdot E_{p}}{r^{3} \cdot\left(\rho_{i} \cdot C_{\mathrm{p}, i}\right)_{\min }},
$$

where $n$ is the number of virtual heat particles currently located at the site. Monte Carlo time $t_{\mathrm{MC}}$ needs to be converted to real time. The method for doing this is described in [17]. The result is:

$$
t=\frac{t^{M C}}{6} \cdot \frac{s^{2}\left(\rho C_{p}\right)_{\min }}{\lambda_{\max }}
$$


As an example of a temperature profile calculated by the LMC method, in Fig. 4 we show the temperature profile in a layered composite of paraffin and aluminium with the layers arranged normal to the heat flow. The thermal parameters of these two phases are of course very different. In the same figure are the results of a determination of the temperature profile using Finite Element analysis. It can be seen that there is excellent agreement between the two methods.

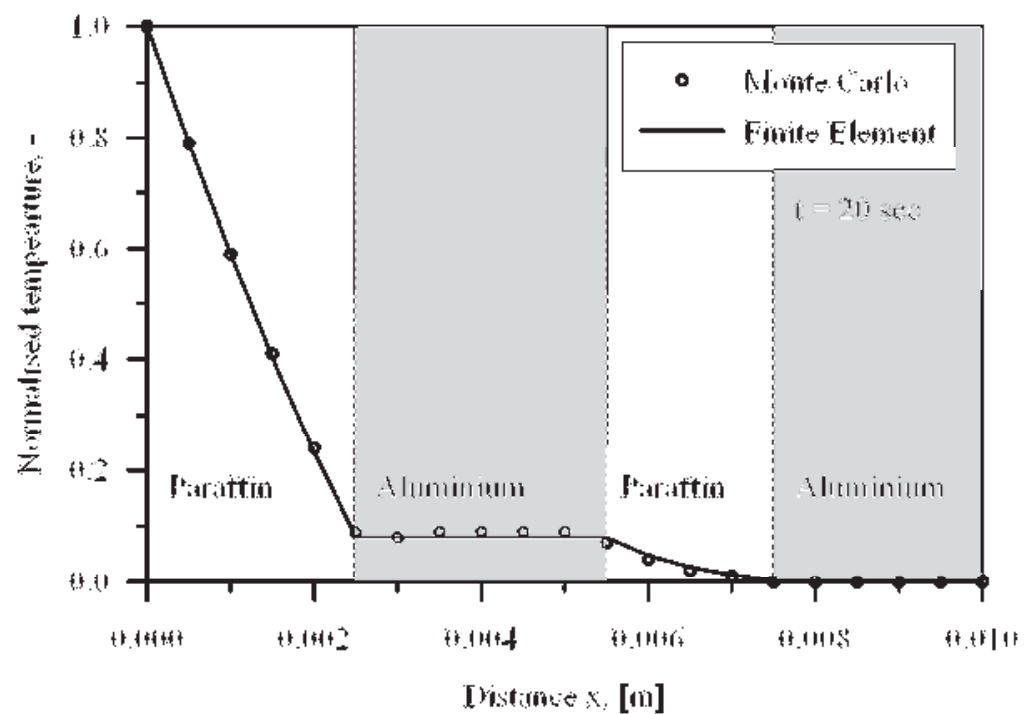

Fig. 4 Simulated temperature profile in a layered two phase paraffin - aluminium composite.

The dependence of the thermal conductivity (and the density and specific heat) on temperature can be included in the calculation of the temperature profiles using an extension of what was achieved with the composition dependence of the diffusivity when determining concentration profiles [18].

\section{Determination of the effective elastic bulk modulus}

This is a very new application of the LMC method and, as a consequence, much development work needs to be done. In isotropic elastic media problems, two moduli are necessary for the determination of the unknown strains/stresses. The same is true for isotropic composite media where two effective moduli are needed. So far, we can calculate by means of LMC (the agreement with a FE method calculation is extremely good) only one effective elastic modulus, namely the bulk modulus $\mathcal{K}_{\text {eff. }}$ To make a full analogy between the effective elastic bulk modulus and the effective mobility/diffusivity of the moving particles in the LMC calculations we establish a simple nexus between the bulk modulus and the jump frequency. Let us consider an isotropic $3 \mathrm{D}$ or $2 \mathrm{D}$ elastic composite media consisting of two phases - the matrix phase and an inclusion phase, where the geometry of the inclusion phase can be approximated by spheres or circles. In the derivation, we use the following elastic properties of the phases: the bulk modulus, the Young's modulus, the Lamé coefficient $\mu$ and the Poisson ratio for the matrix phase $-\mathcal{K}_{\mathrm{m}}, E_{\mathrm{m}}, \mu_{\mathrm{m}}$ and $\nu_{\mathrm{m}}$ and $\mathcal{K}_{\mathrm{i}}$, $E_{\mathrm{i}}, \mu_{\mathrm{i}}$ and $\nu_{\mathrm{i}}$ for the inclusion phase. An analytical expression for the effective bulk modulus for this media has been derived in [19, see also 20] for the 3D case and can 
be generalised into the following form for both 3D $(d=3)$ and 2D $(d=2$, plane stress formulation) cases:

$$
\hat{\mathcal{K}}=\mathcal{K}_{m}+\frac{g\left(\mathcal{K}_{i}-\mathcal{K}_{m}\right)}{1+(1-g)\left(\mathcal{K}_{i}-\mathcal{K}_{m}\right) /\left(\mathcal{K}_{m}+2(d-1) \mu_{m} / d\right)}
$$

where

$$
\mathcal{K}_{m(i)}=\frac{E_{m(i)}}{d\left(1-(d-1) v_{m(i)}\right)}
$$

For plane strain conditions Eq. (14) is still valid with the following expression for $\mathcal{K}_{m(i)}$ :

$$
\mathcal{K}_{m(i)}=\frac{E_{m(i)}}{2\left(1+v_{m(i)}\right)\left(1-2 v_{m(i)}\right)}
$$

In [19] it was shown that Eq. (14) is exact for the 3D case (the 2D case can be proved using analogous reasoning) when the inclusions are well separated from one another. For more realistic cases where the inclusions are close or touching each other, numerical methods are the only means to determine the effective elastic properties of the composite.

To proceed further, we give the Maxwell expression for the effective mass diffusivity in the following form:

$$
\hat{D}=D_{m}+\frac{g\left(D_{i}-D_{m}\right)}{1+(1-g)\left(D_{i}-D_{m}\right) /\left(d D_{m}\right)}
$$

where $D_{\mathrm{m}}$ and $D_{\mathrm{i}}$ are the diffusivities of the matrix and inclusion phases. We present this equation to compare with Eq. (14) for the effective bulk modulus and this comparison will be used in the following discussion/derivation.

To establish the nexus we proceed in the following way. The bulk modulus is responsible for the process of dissipation of elastic energy. This can be observed in practice when it occurs on a large, geotechnical scale. Nevertheless, it means that the process can be represented by a modified random walk where the corresponding effective properties of the media can be calculated using Einstein-type of expressions. The modification of the random walk should reflect the nature of the elastic type of process. Then, in general, it should be essentially a biased random walk, not a simple unbiased random walk that has been considered so far above in the mass diffusion and thermal diffusion sections. Work to resolve this is currently under way.

On the other hand, it should be recalled that we have two remarkably very close analytical expressions (Eq. (14) and Eq.(16)) for these two different random walks! Both these expressions are exact for the idealised composite media. Keeping this in mind, we can try the easiest way to modify the 'simple' random walk (used so far for mass and thermal diffusion) in order to calculate the properties of the 'biased' random walk (for the calculation of the elastic properties). Now, it can be easily shown that $\hat{\mathcal{K}}$ in Eq. (14) can be approximated from above by the Maxwell expression for the effective bulk modulus as follows: 


$$
\hat{\mathcal{K}} \leq \mathcal{K}_{m}+\frac{g\left(\mathcal{K}_{i}-\mathcal{K}_{m}\right)}{1+(1-g)\left(\mathcal{K}_{i}-\mathcal{K}_{m}\right) /\left(d \mathcal{K}_{m}\right)}
$$

a)

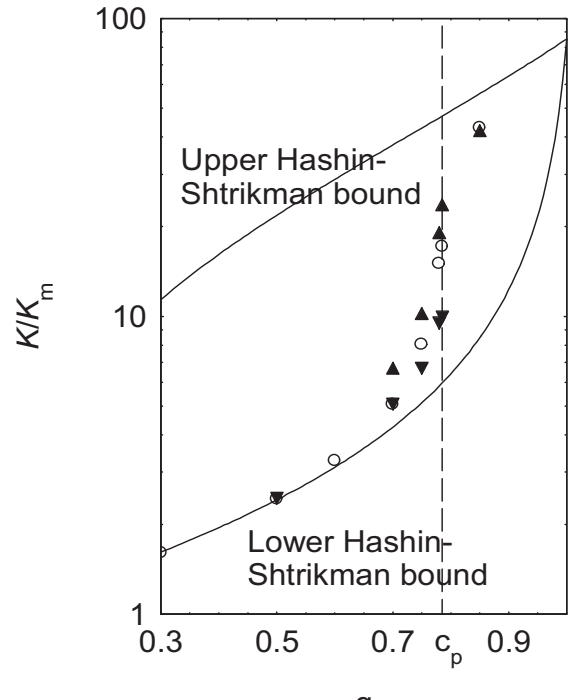

g b)

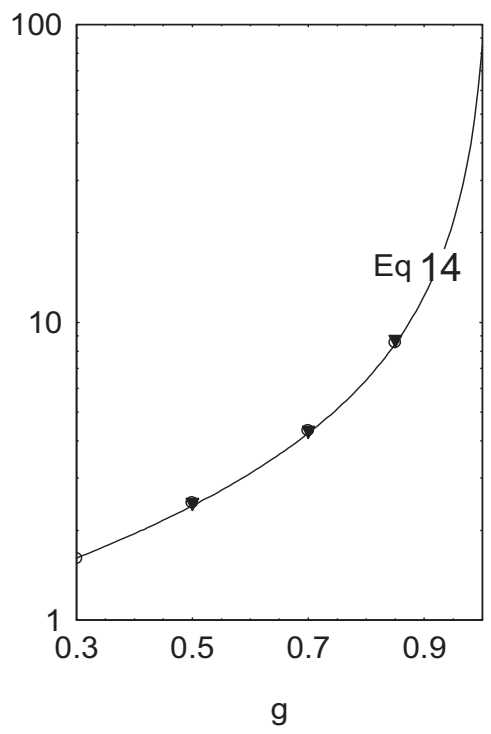

Fig 5. Results of finite element (open circles) and LMC (bold triangles) calculations for the ratio $\mathcal{K} / \mathcal{K}_{\mathrm{m}}$ as a function of the volume fraction of inclusions: a) $2 \mathrm{D}$ calculations for regular circular inclusions; b) 2D calculations for regular square inclusions.

Here the equality sign will always hold if $v_{\mathrm{m}}=0$. This means that for this case $\left(v_{\mathrm{m}}=\right.$ 0 ), $\hat{\mathcal{K}}$ can be calculated exactly by using the standard LMC method when the $\mathcal{K}_{\mathrm{i}}$ and $\mathcal{K}_{\mathrm{m}}$ are simply cast into the form of jump frequencies. For $\nu_{\mathrm{m}} \neq 0$ an easy way to proceed is as follows. For a given volume fraction of inclusions and a given $k=\mathcal{K}_{i} / \mathcal{K}_{m}$, we find the 'adjusted' ratio $k^{\prime}=\mathcal{K}_{i}^{\prime} / \mathcal{K}_{m}$ that will satisfy the equation:

$$
\frac{\hat{\mathcal{K}}}{\mathcal{K}_{m}}=1+\frac{g\left(k^{\prime}-1\right)}{1+(1-g)\left(k^{\prime}-1\right) / d},
$$

where $\hat{\mathcal{K}}$ is given by Eq. (14). This equation is easy to solve and the resulting ratio can be used for LMC simulations (see Fig. 5). In the same figure we plot the wellknown upper and lower Hashin-Shtrikman bounds [19] for comparison. We note that the LMC simulations are in a perfect agreement with FE calculations for square inclusions and in a good agreement for the circular inclusions. This is perhaps not a surprising result given that the bulk modulus is remarkably isotropic for a wide range of composite materials and this appears to make the application of the LMC method quite straightforward.

\section{Acknowledgments}

Research support from the Australian Research Council is gratefully acknowledged.

\section{References}

[1] P. Benoist and G. Martin, Thin Solid Films Vol. 25 (1975), p.181. 
[2] G.E. Murch, Diffusion and Defect Data, Vol. 32 (1983), p.1.

[3] L.G. Harrison, Trans. Farad. Soc. Vol. 57 (1961), p.1191.

[4] I. Stloukal and J. Cermak, Defect Diffus. Forum Vol. 263 (2007), p.189.

[5] E.W. Hart, Acta Met. Vol. 5 (1957), p.597.

[6] I. Kaur, Y. Mishin and W. Gust, Fundamentals of Grain and Interphase Boundary Diffusion, Wiley, Chichester, 1995.

[7] I.V. Belova and G.E. Murch, Defect Diffus. Forum Vol. 261/262 (2007), p.103.

[8] I.V. Belova and G.E. Murch, Phil. Mag. Vol. 84 (2004), p.17.

[9] I.V. Belova and G.E. Murch, J. Phys. Chem. Solids Vol. 66 (2005), p.722.

[10] I.V. Belova and G.E. Murch, J. Meta. Nanocryst. Mat. Vol. 19 (2004), p.23.

[11] I.V. Belova and G.E. Murch, Phil. Mag. A Vol. 81 (2001), p.2447.

[12] S.V. Divinski, F. Hisker, Y.-S. Kang, J.-S. Lee and Chr. Herzig, Z. Metallk. Vol. 93 (2002), p.256.

[13] I.V. Belova and G.E. Murch, J. Mat. Proc. Tech. Vol. 153/154 (2004), p.741.

[14] T. Fiedler, A. Öchsner, N. Muthubandara, I.V. Belova and G.E. Murch, Mat. Sc. Forum, Vol. 553 (2007), p.51.

[15] T. Fiedler, A. Öchsner, I.V. Belova and G.E. Murch, Defect Diffus. Forum, Vol. 273/276 (2008), p.216.

[16] T. Fiedler, A. Öchsner, I.V. Belova and G.E. Murch, Defect Diffus. Forum, Vol. 273/276 (2008), p.222.

[17] I.V. Belova, G.E. Murch, T. Fiedler and A. Öchsner, Diffusion Fundamentals, Vol. 4 (2007), p.15.1.

[18] Unpublished results of the authors.

[19] Z. Hashin and S. Shtrikman, J. Mech. Phys. Solids Vol. 11 (1963), p.127.

[20] R.M. Christensen, Mechanics of Composite Materials, John Wiley \& Sons, 1979. 
Diffusion in Solids and Liquids IV

doi:10.4028/www.scientific.net/DDF.283-286

Lattice-Based Walks and the Monte Carlo Method for Addressing Mass, Thermal and Elasticity Problems

doi:10.4028/www.scientific.net/DDF.283-286.13 